\section{A Time-symmetric Hubble-like Law: Light Rays Grazing Randomly Moving Galaxies Show Distance-Proportional Redshift}

\author{
O. E. Rössler, D. Fröhlich, and N. Kleiner
}

Division of Theoretical Chemistry, University of Tübingen, 72076 Tübingen, Germany

Reprint requests to Prof. O. E. R.; Fax: 07071/296910

Z. Naturforsch. 58a, $807-809$ (2003);

received November 24, 2003

A new class of dissipative structures is proposed that live in real space rather than phase space. A light ray passing through a soup of randomly moving gravitating masses is a case in point. It suffers a "dynamic path elongation" since the random pushes and pulls have a greater probability of increasing than decreasing its path length. Time reversal then re-shrinks the path in question in a conspirational manner, while a close-by nonselected path gets expanded. This is a new statistical-mechanics phenomenon. The latter at the same time qualitatively reproduces the well-known Hubble phenomenon of distance-proportional light-path expansion in the cosmos. A preliminary quantitative estimate, based on the Birkinshaw equation with an assumed bias factor of three, is also presented.

Key words: Hubble Law; Time Reversibility; Chaotic Billiards; Fermi Deceleration; Stirred Lentils' Soup.

The famous Hubble phenomenon of a distanceproportional cosmic redshift apparently possesses an analog that arises without any special assumptions. An average light ray passing through the cosmos is lined by about 5000 nearest galaxies that lie within a range of one million light years or ten galactic diameters each and move in a random direction at 0.1 to 1 percent the speed of light. (Note that 5000 is the approximate cubic root of $10^{11}$, the current number of visible galaxies, and that the mean diameter of a tube containing 5000 galaxies in a cosmos 10 billion light years across is 2 million light years.) Such a statisticalmechanical scenario appears unfamiliar. What are the implications?

Each galaxy slightly curves the adjacent light path and, when in motion, slightly dynamically increases or decreases its length. This fact is well known [1]. While individual path-shortening and path-elongating trans- verse galactic motions are equally effective in their blueshift and redshift generating properties [2], their concatenation shows a bias. The intuitive reason is that a path that is almost straight is "harder to make more straight than less straight." Dynamic path elongation is favored over dynamic path shortening because the random "baggying influences" exerted by the moving gravitational lenses are unequally likely to elongate and to shorten the light path.

The new prediction immediately implies that in the negative direction of time, the path magically "unwiggles" (suffers a decrease in bagginess and length). This is puzzling since the perturbing galactic motions remain randomly distributed under negative time: hence an increase of wiggledness should occur also in negative time. The contradiction disappears if one realizes that there exists a difference between the positive and the negative time direction in the present case: The more wiggled light path produced in the positive direction of time is already correlated with the random galactic motions that lie in its future in negative time. Hence the latter motions are no longer random with respect to this particular light ray.

However, such an exact time reversal is only half the story. If the incoming light ray is, in the negative direction of time, replaced by a slightly perturbed light ray, the latter will, after a transient period of "unwiggling," start veering away from the former in chaotic divergence in order to embark on a wiggling-type career of its own. This behavior in real space is reminiscent of a familiar situation in phase space: When a gas is released in its phase space from a far-fromequilibrium initial condition, a time reversal performed after a finite time of forward evolution likewise produces a rare "anti-dissipative path" only in the total absence of any perturbation. Otherwise, its entropy soon ceases to go down further in order to re-embark on an uphill course - just as it did in the original direction of time [3]. The present prediction of an analogous phenomenon arising in real space nonetheless comes as a surprise.

An estimate of the size of the anticipated effect can be obtained by starting out from Birkinshaw's equation [2]

$$
z=a \cdot \frac{v}{c} \cdot \frac{R_{\mathrm{S}}}{R}
$$


This equation describes the redshift (positive values of $z$ ) or blueshift (negative values of $z$ ) caused by a transversely moving lens. The positive sign of the lens's velocity $v$ (and hence $z$ ) applies if the lens is approaching the transversely passing light ray. One sees that $z$ depends linearly, apart from $v$, on $R_{\mathrm{S}}$ (the Schwarzschild radius of the lens and hence its mass) and is thirdly inversely proportional to the minimum passing distance, $R$. The remaining constant factor, $a$, can be estimated to be $\approx 6$ from a realistic example (a galactic cluster of $10^{15}$ solar masses traveling at $v=0.002 c$ ) presented by Aghanim et al. [4] (see their Fig. 1).

Equation (1) is perfectly symmetric as far as the generation of a redshift or blueshift is concerned. However, if two parallel photons are considered that both graze the same transversely moving lens (one in front, the other in the back) while leaving the lens with a zero combined change of its angular momentum, a factor-3 bias in favor of redshift is found [5]. If we assume this bias factor to be representative, (1) can be used to calculate the cumulative effect valid after many encounters, by simply adding up the individual effects. Let us briefly look at such a calculation.

There are (as we saw) 5000 nearest galaxies to be encountered by an average light ray over a distance of $10^{10}$ light years. In addition, there also lie 500 encounters on its way with clusters of a thousand galaxies, 50 encounters with superclusters of a million galaxies, and 5 encounters with megaclusters of a billion galaxies (if a condition of maximum statistical homogeneity is assumed for simplicity's sake). Equation (1), with $a=4$ (mean of +6 and -2 ) then yields the following result: The individual galaxies encountered have an average ratio $R_{\mathrm{S}} / R$ of 0.03 light years (the Schwarzschild radius of the $10^{11}$ suns contained in a galaxy) divided by one million light years, i.e. $R_{\mathrm{S}} / R=3 \cdot 10^{-8}$. Taken together with $a=4, v=0.003 c$ and $N=5000$, this value yields a cumulative redshift of $z=2 \cdot 10^{-6}$, a small number indeed.

Enter the next levels of the hierarchy (clusters, superclusters, etc.). Each level adds a contribution that exceds the preceding one by a factor of 10 (since the mass-increase by a factor of 1000 for $R_{\mathrm{S}}$ is partially offset by a tenfold increase of $R$ and a tenfold reduction of $N$ ). This pushes the cumulative redshift up by three orders of magnitude, to $z=2 \cdot 10^{-3}$. This result is still by a factor of 1000 too low to accomodate the empirical Hubble redshift. However, inclusion of dark matter (omitted so far) will add a factor of 50 while admission of marked deviations from statistical homogeneity (assumed so far) can add a factor of ten. The final estimate thus obtained, $z=1$, appears "suggestive."

However, it all depends on the assumed bias factor of 3 as mentioned. While the existence of a bias factor greater than unity follows from the above "baggying" argument (and the special example considered in [5]), it also follows from recent numerical simulations of "Fermi deceleration" in a chaotic billiard subjected to periodically or randomly moving boundaries [6]. Since Loskutov, Ryabov, and Akinshin did not include attracting moving boundaries (which behave analogously in the grazing case) in their simulations, they did not yet draw the connection to the cosmos. Their result nevertheless amounts to a "numerical existence proof" for a bias factor greater than unity - the main proposal made above.

To conclude, a qualitatively new statisticalmechanical effect was suggested: a bias in favor of dynamic light-path elongation in a cauldron of randomly moving gravitational lenses (a "stirred lentils' soup," lentil meaning lens). The effect has the attractive property of being valid in either direction of time. No entropy-analogous effect appears to have been proposed in cosmology before. The entropic effect on light, if confirmed, could relieve the only known fundamental law of nature, that is non-timereversal invariant (the Hubble law), from its odd status. Loskutov-type simulations can be used to determine the exact magnitude of the effect.

\section{Acknowledgements}

We thank the late Ilya Prigogine for a stimulating exchange. We also thank André K.T. Assis, Sebastian Fischer, Joachim Peinke, Jürgen Parisi, Wolfgang Engelmann, Wolf Koch, Claus Kahlert, Dietrich Hoffmann, Christian Ochsenfeld, Rudolf Hübener, Thilo Hinterberger, Florian Grond, Anthony Moore, Peter Bosetti, Bruno Eckhardt, Roger Malina, George Lasker, Christophe Letellier, Daniel Dubois, Laurent Nottale, Peter Weibel, Stefan C. Müller, Achim Kittel, and A. Hilfinger for discussions. An anonymous referee kindly drew our attention to reference 4 . For J. O.R. 
[1] L. Nottale and J. P. Vigier, Continuous increase of Hubble modulus behind clusters of galaxies. Nature London 268, 608 (1977).

[2] M. Birkinshaw, Moving gravitational lenses. In: Gravitational Lenses, ed. by J. M. Moran, J. N. Hewitt, and K. Y. Lo, Springer Verlag, Berlin, Lecture Notes in Physics 330, 59-64 (1988).

[3] I. Prigogine, E. Kestemont, and M. Mareschal, Velocity correlations and irreversibility. In: From Chemical to Biological Organization, ed. by M. Markus, S.C. Müller, and G. Nicolis, pp. 22-26, Springer-Verlag, Berlin 1988.
[4] N. Aghanim, S. Prunet, O. Forni, and F. R. Bouchet, Moving gravitational lenses: imprints on the cosmic microwave background. Astron. Astrophys. 334, $409-$ 419 (1998).

[5] O. E. Rössler and C. Letellier, Fermi deceleration reencountered: Recurrent grazing-type collisions with supermassive slow particles imply distance-proportional cooling (Abstract). Rouen Chaos Conference, December 15, 2003.

[6] A. Loskutov, A. B. Ryabov, and L. B. Akinshin, Analysis of billiards with time-dependent boundaries. Facta Universitatis, Series Mechanics, Automatic Control and Robotics 3, 99-116 (2001). 